\title{
S-I-3. The Posteromedial Hypothalamotomy for the Treatment of Behavior Disorders
}

\author{
Hiroaki SEkIno \\ Department of Neurosurgery, Faculty of Medicine \\ University of Tokyo, Tokyo
}

Autonomic and somatomotor responses upon stimulation of the posteromedial part of the hypothalamus were reported in 55 patients with aggressive or violent behavior and restlessness. The coordinates of the target points were $1 \mathrm{~mm}$ anterior to $3 \mathrm{~mm}$ posterior to the midpoint of the intercommissural line, 2 to $4 \mathrm{~mm}$ inferior to the intercommissural line and $2 \mathrm{~mm}$ lateral to the lateral wall of the third ventricle. Stimulation of the points mentioned above, caused rise in blood pressure, tachycardia, tachypnea and maximal dilatation of pupilles. In the same time, somatomotor responses were induced; namely, the head of the patinet tilted toward the side of stimulation and the ipsilateral eye moved to medioinferior direction. Electroencephalographically, desynchronization or diffuse irregular delta waves of high voltage were induced in all leads and continued several minutes after the cessation of the stimulation. Points where sympathetic reactions were most marked, were electrocauterized bilaterally. During the follow-up period of more than 2 years, this operation produced marked calm effects, namely, 43 cases out of followed up 46 cases remained in good results.

Cases with violent behavior showed higher plasma levels of nonesterified fatty acids (NEFA). These were markedly elevated by electrical stimulation of the posteromedial hypothalamus and after destruction plasma levels of NEFA decreased in normal level.

\section{S-I-4. Psychosurgical Methods from Psychiatry}

\author{
Sadao Hirose \\ Department of Neuropsychiatry, Nippon Medical School, Tokyo
}

Over thirty five years have elapsed since psychosurgery was hailed as an epochmaking somatic treatment in psychiatry. But marked decline in its popularity followed because the frequent misuse of the extensive prefrontal lobotomy for many deteriorating schizophrenics yielded unsatisfactory results. The introduction of the psychotropic drugs increased this tendency. Some psychiatrists, especially in Japan, have been critical and over-sceptical of the psychosurgical approach to relieve the most tortured psychiatric patients. However, psychosurgery still has a definite application in certain well-selected cases. Choice of patients is the vital factor. Choice 
of target is also important. The old standard lobotomy has been replaced by limited selective operations, including stereoencephalotomy. The clinical picture and the premorbid personality traits of the patient are most important factors in the selection of patients.

The author employed various psychosurgical operations on 522 patients between the years 1947 and 1971. A new method of orbito-ventromedial undercutting (Hirose) was begun in July 1957, and used in 122 cases. The last few years I have been doing several operations per year after strict psychiatric case selection by myself as a psychiatrist and our neuropsychiatric staff. The results are excellent. Most satisfactory results have been obtained in affective psychoses, such as chronic protracted depressive state, especially involutional melancholia, with severe insomnia, anxiety, suicidal attempts and various intractable physical complaints. Involutional paranoid state with affective coloring also responded favorably. Obsessive compulsive neurosis and anxiety neurosis showed good results. Favorable outcome were also obtained in atypical schizophrenia with well-preserved personality with recurrent catatonic episodes, schizo-affective type, and chronic neurosis-like state. Epilepsy and postencephalitic behavior disorder with explosive tendency were also benefited by the orbito-ventromedial undercutting. The contraindications for the operation will be deteriorated schizophrenia, severe mental deficiency, personality disorders with shiftlessness (Haltlosigkeit), irresponsibility, addiction, criminal tendency for theft and callous emotionality. Orbitoventromedial undercutting is recommended for such psychiatric states as protracted emotional tension states, oversensitivity, excessive self-consciousness, and obsessional states. The premorbid personality traits of the patients with favorable results include delicate, warm-hearted, conscientious, sensitive, enthusiastic, anankastic personalities. In short, they are socially active personalities. The premorbid personality traits of the patients with unfavorable results include asocial, callous, unfeeling, gloomy, shiftless, irresponsible, immature, lonely and autistic personalities. Orbito-ventromedial undercutting was found to produce the maximum therapeutic effect on the emotional aspects of the mental disorder without serious post-operative complications and impairment of personality. Freeman stressed the importance of interrupting the fasciculus uncinatus in the psychosurgical operations. It was verified that my orbito-ventromedial undercutting involved the fasciculus uncinatus by the brain of two patients.

Finally, I should like to suggest the necessity of much more closer cooperation between the neurosurgeon and the psychiatrist in the future. 